\title{
One-loop Dirac neutrino mass and mixed axion-WIMP dark matter
}

\author{
Cristian D. R. Carvajal ${ }^{*}$ and Óscar Zapata ${ }^{\dagger}$ \\ Instituto de Física, Universidad de Antioquia, Calle 70 No. 52-21, Medellín, Colombia
}

(Received 14 January 2019; published 9 April 2019)

\begin{abstract}
We consider the Peccei-Quinn (PQ) mechanism as the one behind the Dirac neutrino masses when these are generated through the $d=5$ effective operator $\bar{L} \tilde{H} N_{R} \phi$ at one-loop level, with $\phi$ being a Standard Model singlet scalar. In this setup, the PQ symmetry guarantees that the one-loop realization of such an effective operator gives the leading contribution to the Dirac neutrino masses by forbidding the contributions arising from its tree-level realizations. All the mediators in the one-loop neutrino mass diagrams can be stabilized by a remnant $Z_{N}$ symmetry from the PQ symmetry breaking, thus forming a dark sector besides the axion sector and leading to mixed axion-WIMP dark matter scenarios.
\end{abstract}

DOI: 10.1103/PhysRevD.99.075009

\section{INTRODUCTION}

In the last decades the neutrino oscillations have been firmly established thanks to a great dedicated experimental program [1], leading to a concise and a clear understanding of the neutrino oscillation pattern [2]. However, this wisdom has not shed light on the underlying mechanism behind the neutrino masses and the properties of neutrinos under the particle-antiparticle conjugation operation. This adds to the negative results regarding the Majorana nature of the neutrinos from neutrinoless double-beta decay experiments [3-8]. Hence, a growing interest in Dirac neutrinos mass models has recently appeared, especially in models that contain a weakly interacting massive particle (WIMP) [9] as the dark matter candidate that is not trivially connected with the neutrino mass generation mechanism [10-24].

It worth noting here that the main motivation for considering WIMPs is that they lie at the scale at which the physics beyond the Standard Model (SM) is expected to appear, thus leading to signals in dedicated experiments looking for dark matter (DM). However, the WIMP paradigm is not free of theoretical and experimental challenges [25], such as the lack of signals in those experiments so far, which have led to severe constraints over the parameter space of WIMP models [25-28]. Therefore, it would be relevant to consider approaches

\footnotetext{
*david.ruiz@udea.edu.co

†oalberto.zapata@udea.edu.co
}

Published by the American Physical Society under the terms of the Creative Commons Attribution 4.0 International license. Further distribution of this work must maintain attribution to the author(s) and the published article's title, journal citation, and DOI. Funded by SCOAP . beyond the WIMP paradigm such as the multicomponent DM [29,30], where the DM of the Universe is composed by, for instance, WIMPs and QCD axions [31-36].

Within the framework of the SM it is not possible to form a Dirac mass term for the neutrinos because of the lack of right-handed partners of the active neutrinos $\nu_{L}$. Once they are added, Dirac neutrino masses are generated via the $d=4$ operator

$$
\mathcal{O}_{4}=y \bar{L} \tilde{H} N_{R}+\text { H.c. }
$$

where $N_{R}$ are right-handed neutrinos, $L=\left(\nu_{L}, \ell_{L}\right)^{T}$ is the left-handed lepton doublet, and $H=\left(H^{+}, H^{0}\right)^{T}$ is the Higgs doublet. Under the lepton number conservation (to protect the Diracness of neutrinos), this operator leads to sub-eV neutrino masses for $|y| \lesssim 10^{-13}$, thus leaving unsettled the explanation of the smallness of the neutrino mass scale. From this, it would be reasonable to forbid $\mathcal{O}_{4}$ through a certain symmetry $\mathcal{X}$, while generating Dirac neutrino masses via higher dimensional operators, either at tree or loop level. On those lines, if the Higgs doublet is the only scalar in the particle spectrum, the lowest dimensional operator would be of dimension $d=6$, while if a scalar SM singlet $\phi$ charged under $\mathcal{X}$ is added, then a $d=5$ operator $\mathcal{O}_{5}$ would arise [14,21,24,37]

$$
\mathcal{O}_{5}=y^{\prime} \bar{L} \tilde{H} N_{R} \phi+\text { H.c. }
$$

It follows that after the spontaneous breaking of $\mathcal{X}$ (due to the vacuum expectation value of $\phi$ ) an effective $\mathcal{O}_{4}$-like operator will arise but with the benefit that $\mathcal{O}_{4}$ itself is forbidden. Additionally, the $\mathcal{X}$ symmetry may also may serve as the stabilizing symmetry of possible WIMP candidates arising from the one-loop (ultraviolet) realizations of $\mathcal{O}_{5}[13-19]$. 
In this work, we associate the Peccei-Quinn (PQ) mechanism [38] to scotogenic models with one-loop Dirac neutrino masses ${ }^{1}$ through identification of $\phi$ in $\mathcal{O}_{5}$ as the scalar field hosting the QCD axion and the PQ symmetry as responsible for the absence of $\mathcal{O}_{4}$ and the stability of the possible WIMP candidates. It follows that after the spontaneous electroweak and PQ symmetry breaking Dirac neutrino masses will be generated via the $\mathcal{O}_{5}$ operator, and imposing that $H$ does not carry a PQ charge (while $L$ and/or $N_{R}$ do) the contribution to the neutrino masses from the $\mathcal{O}_{4}$ operator is automatically forbidden. Under some PQ charge assignments, it is also possible to make the one-loop realization of $\mathcal{O}_{5}$ the main source of neutrino masses. That is, the contributions of the three Dirac seesaw mechanisms are no longer present and the smallness of neutrino masses is related to the radiative character besides the large mass suppression coming from the loop mediators. Furthermore, we will show that the same PQ charge assignments lead to a residual $Z_{N}$ discrete symmetry after the PQ symmetry breaking that guarantees the stability of the lightest of the mediators in the one-loop neutrino mass diagrams, thus leading naturally to axionWIMP DM scenarios.

\section{FRAMEWORK}

The apparent nonobservation of $C P$ violation originating from the $\theta$ term in the QCD Lagrangian is a strong theoretical motivation for going beyond the SM, since it can be dynamically explained through the PQ mechanism [38]. Such a mechanism requires one to extend the SM gauge group with an anomalous global symmetry, $U(1)_{\mathrm{PQ}}$, which is spontaneously broken at some high scale [55-58] by the vacuum expectation value $v_{S}$ of the scalar $S$ that hosts the QCD axion $a(x)[59,60]$,

$$
S=\frac{1}{\sqrt{2}}\left[\rho(x)+v_{S}\right] e^{i a(x) / v_{S}} .
$$

Here $\rho(x)$ is the radial part that will gain a mass of order of the PQ symmetry breaking scale, which is constrained by several astrophysical phenomena, such as supernova cooling [61] and black hole superradiance [62] to $v_{S} \sim\left[10^{9}, 10^{17}\right] \mathrm{GeV}$. As a canonical axion model, we consider a hadronic KimShifman-Vainshtein-Zakharov (KSVZ)-type model [55,56], that is, we add to the SM the axionic field $S$ and two chiral singlet quarks $D_{L}$ and $D_{R}$, all of them charged under the PQ symmetry and interacting through the Yukawa term $y_{D} S \overline{D_{L}} D_{R}$.

In the present framework $S$ additionally plays the role of $\phi$ in the $\mathcal{O}_{5}$ operator, with both $L$ and $N_{R}$ charged

\footnotetext{
${ }^{1}$ See Refs. [33,35,39-54] for scenarios where the PQ mechanism is deeply related to the neutrino mass generation (at tree or loop level).
}

under the PQ symmetry, whereas $H$ does not, in such a way $\mathcal{O}_{5}$ is allowed and $\mathcal{O}_{4}$ is banned. Contrary to the original KSVZ model, where all the SM fermions are neutral under the PQ symmetry, in this framework the leptons do have PQ charges but the quarks remain neutral. The lepton number L conservation is further imposed with the usual assignment for the SM fields (one for the leptons and zero for the rest), one for $N_{R}$ and zero for $S$, in order to prevent the appearance of the Majorana mass term $N_{R} N_{R}$ and the $d=5$ Weinberg operator $L H L H$, along their induced partners $N_{R} N_{R} S$ and LHLHS, and thus protecting the Dirac nature of neutrinos. However, it should be noted that by allowing large values for the PQ charges and not imposing $\mathrm{L}$ conservation is possible to obtain a consistent model where the Diracness of neutrinos is guaranteed [47] (see Sec. IV for a specific example).

More specifically, the $S, H$, and $N_{R}$ fields transform under the PQ symmetry as

$$
\begin{aligned}
S & \rightarrow e^{i \mathcal{X}_{S} \xi} S, \quad H \rightarrow H, \\
N_{R} & \rightarrow e^{i \mathcal{X}_{N_{R}} \xi} N_{R}, \quad \text { with } \quad \mathcal{X}_{S} \neq 0 .
\end{aligned}
$$

It follows that the PQ charge of the lepton doublet must be $\mathcal{X}_{L}=\mathcal{X}_{N_{R}}+\mathcal{X}_{S}$ in order to simultaneously make the $\mathcal{O}_{5}$ operator PQ invariant and $\mathcal{O}_{4}$ forbidden. Regarding the PQ charges of $D_{R}$ and $D_{L}$, they are related through the Yukawa term $y_{D} S \overline{D_{L}} D_{R}$, so $\mathcal{X}_{D_{L}}=\mathcal{X}_{D_{R}}+\mathcal{X}_{S}$. This, in turn, implies that the color anomaly coefficient $\mathcal{C}$ of the resulting axion models is set by $\mathcal{C}=\left|\mathcal{X}_{S}\right| / 2=M$, with $M$ a positive integer, ${ }^{2}$ i.e., the PQ charge of $S$ must be even. On the other hand, we assume $\mathcal{X}_{D_{R}} \neq 0$ to avoid the mixing term $\overline{q_{L}} H D_{R}$ [the SM quark sector is not charged under the $\left.U(1)_{\mathrm{PQ}}\right]$. All in all, these $\mathrm{L}$ and PQ charge assignments automatically forbid $\mathcal{O}_{4}$ and the Majorana mass terms for $N_{R}$ and $\nu_{L}$, and leave invariant $\mathcal{O}_{5}$. However, they do not necessarily prevent the contributions to the neutrino mass matrix arising from the tree-level realizations of $\mathcal{O}_{5}$.

In the presence of right-handed neutrinos and a scalar singlet, the tree-level realizations of $\mathcal{O}_{5}$ demand the introduction of either a SM singlet vectorlike fermion, an extra Higgs doublet, or a vectorlike lepton doublet as mediator fields, which lead to the three Dirac seesaw

\footnotetext{
${ }^{2}$ The color anomaly is given by $\mathcal{C}=\sum_{i}\left|\mathcal{X}_{D}-\mathcal{X}_{D_{R}}\right| T\left(\mathcal{C}_{i}\right)$, where the sum is over all the irreducible $S U(3)_{C} \times U(1)_{\mathrm{EM}}$ representations and $T\left(\mathcal{C}_{i}\right)$ is the index of the $S U(3)_{C}$ representation of the $D_{L, R}$ fields $[T(3)=1 / 2]$ [63]. Note that the condition of $\mathcal{C}$ to be an integer is mandatory to guarantee the periodicity of the axion potential, $2 \pi f_{a}=2 \pi v_{S} / \mathcal{C}$, and the interpretation of the axion as the phase of the field $S$ (implying a periodicity of $2 \pi v_{S}$ ) [64,65]. Notice here that $v_{S}$ is related to the axion decay constant $f_{a}$ via $f_{a}=v_{S} / \mathcal{C}$, where the color anomaly also sets the number of vacua of the $S$ (the so-called domain wall number [64]).
} 


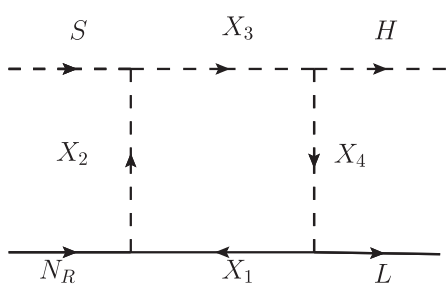

(a) T1-1-A model

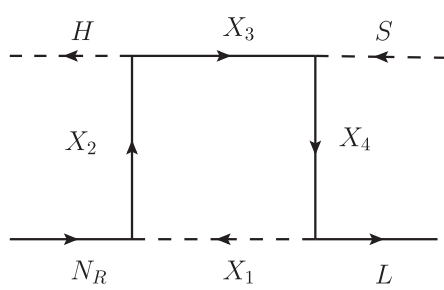

(d) T1-2-B model

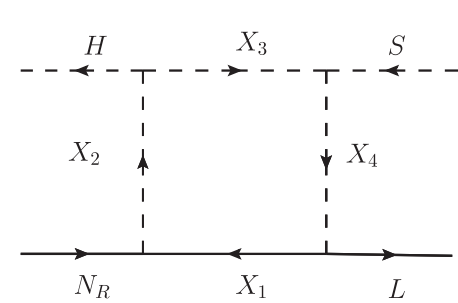

(b) T1-1-B model

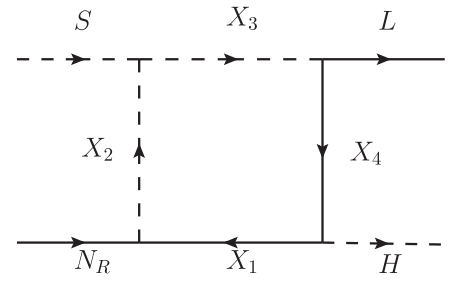

(e) T1-3-D model

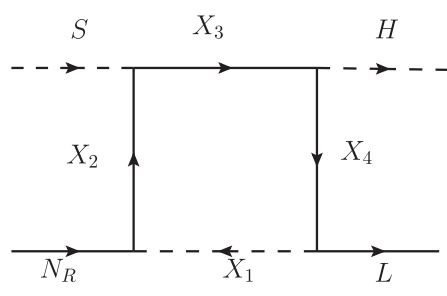

(c) T1-2-A model

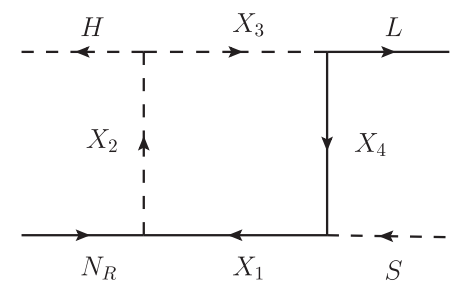

(f) T1-3-E model

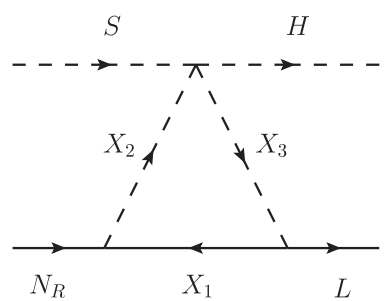

(g) T3-1-A model

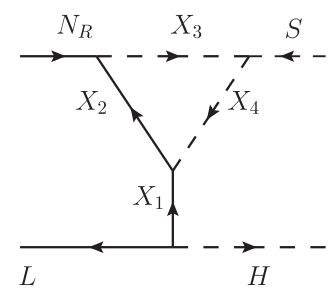

(h) T4-3-I model

FIG. 1. One-loop diagrams with T1, T3, and T4 topologies leading to Dirac neutrino masses.

mechanisms [14,21,24,37]: types I-III, respectively. On the other hand, all possible realizations-considering new fields (fermions and scalars) transforming as singlets, doublets, or triplets under $S U(2)_{L}$, and color singlets, with arbitrary values of the hypercharge but fixed up to a free parameter $\alpha^{3}$ - of $\mathcal{O}_{5}$ leading to Dirac neutrino masses at one-loop level were studied in Ref. [21]. There it was obtained that a finite list of sets of mediator fields exists, which give rise to neutrino masses at one-loop level, classified according to the topology of the diagram. In that analysis, a $Z_{2}$ symmetry was used to forbid $\mathcal{O}_{4}$, with both $N_{R}$ and $S$ odd and the SM sector even under this $Z_{2}$, and the new fermions were assumed to be vectorlike to ensure anomaly cancellation of the SM gauge symmetries. We will use their results regarding the renormalizable and genuine one-loop models to study the necessary conditions

\footnotetext{
${ }^{3}$ The hypercharge of the mediators is set to $Y=\alpha$ or $Y=$ $\alpha \pm 1$ so that the possible values for $\alpha$ are $0,1,2$ in order to have $S U(2)_{L}$ multiplets featuring a neutral particle.
}

to implement the PQ mechanism as the responsible behind the one-loop Dirac neutrino masses. ${ }^{4}$

At one-loop level, there are three different topologies that lead to renormalizable and genuine one-loop realizations [21]: the T1 (boxlike), T3 (trianglelike), and T4 (penguinlike) topologies. For $\mathrm{T} 1$ and $\mathrm{T} 4$ topologies, there exist different possibilities of assigning the fields in $\mathcal{O}_{5}$ to the four external legs, hence several one-loop diagrams appear (see Fig. 1). The mediator fields in all the one-loop diagrams are denoted by $X_{i}$, where $i$ runs from 1 to 3(4) for the T3 topology (T1 and T4 topologies) and denotes the number of mediators. The Lorentz nature, scalar or fermion, is shown in the corresponding one-loop diagram by a dashed or solid line, respectively.

In Table I we show the $\mathrm{L}$ and PQ charges of the mediator fields that are consistent with our assumptions: (i) axionic one-loop realizations of the $\mathcal{O}_{5}$ operator and prohibition of the $\mathcal{O}_{4}$ operator, (ii) lepton number conservation,

\footnotetext{
${ }^{4}$ We will closely follow the notation introduced in Ref. [21] for the topologies, fields, and genuine models.
} 
TABLE I. L and PQ charges of the mediator fields $X_{i}$ of T1, T3, and T4 models. $\delta, \mathcal{X}_{X_{1}}$, and $\mathcal{X}_{X_{4}}$ are free real parameters, whereas $\mathcal{X}_{S}$ and $\mathcal{X}_{N_{R}}$ denote the PQ charges of $S$ and $N_{R}$, respectively. The Lorentz nature of $X_{i}$ is inferred from the corresponding one-loop diagram (see text for details).

\begin{tabular}{|c|c|c|c|c|c|}
\hline Model & Symmetry & $X_{1}$ & $X_{2}$ & $X_{3}$ & $X_{4}$ \\
\hline $\mathrm{T} 1-(1 / 2)-\mathrm{A}$ & $\begin{array}{c}U(1)_{\mathrm{L}} \\
U(1)_{\mathrm{PQ}}\end{array}$ & $\begin{array}{c}\delta-1 \\
\mathcal{X}_{X_{4}}-\left(\mathcal{X}_{N_{R}}+\mathcal{X}_{S}\right)\end{array}$ & $\mathcal{X}_{X_{4}}{ }^{\delta}-\mathcal{X}_{S}$ & $\begin{array}{c}\delta \\
\mathcal{X}_{X_{4}}\end{array}$ & $\begin{array}{c}\delta \\
\mathcal{X}_{X_{4}}\end{array}$ \\
\hline $\mathrm{T} 1-(1 / 2)-\mathrm{B}$ & $\begin{array}{c}U(1)_{\mathrm{L}} \\
U(1)_{\mathrm{PQ}}\end{array}$ & $\mathcal{X}_{X_{4}}-\left(\mathcal{X}_{N_{R}}+\mathcal{X}_{S}\right)$ & $\mathcal{X}_{X_{4}}-\mathcal{X}_{S}$ & $\mathcal{X}_{X_{4}}{ }^{\delta}-\mathcal{X}_{S}$ & $\stackrel{\delta}{\mathcal{X}_{X_{4}}}$ \\
\hline T1-3-D & $\begin{array}{l}U(1)_{\mathrm{L}} \\
U(1)_{\mathrm{PQ}}\end{array}$ & $\begin{array}{c}\delta \\
\mathcal{X}_{X_{4}}\end{array}$ & $\begin{array}{c}\delta+1 \\
\mathcal{X}_{N_{R}}+\mathcal{X}_{X_{4}}\end{array}$ & $\begin{array}{c}\delta+1 \\
\mathcal{X}_{N_{R}}+\mathcal{X}_{X_{4}}+\mathcal{X}_{S}\end{array}$ & $\begin{array}{c}\delta \\
\mathcal{X}_{X_{4}}\end{array}$ \\
\hline T1-3-E & $\begin{array}{l}U(1)_{\mathrm{L}} \\
U(1)_{\mathrm{PQ}}\end{array}$ & $\mathcal{X}_{X_{4}}+\mathcal{X}_{S}$ & $\begin{array}{c}\delta+1 \\
\mathcal{X}_{N_{R}}+\mathcal{X}_{X_{4}}+\mathcal{X}_{S}\end{array}$ & $\begin{array}{c}\delta+1 \\
\mathcal{X}_{N_{R}}+\mathcal{X}_{X_{4}}+\mathcal{X}_{S}\end{array}$ & $\begin{array}{c}\delta \\
\mathcal{X}_{X_{4}}\end{array}$ \\
\hline T3-1-A & $\begin{array}{l}U(1)_{\mathrm{L}} \\
U(1)_{\mathrm{PQ}}\end{array}$ & $\begin{array}{c}\delta \\
\mathcal{X}_{X_{1}}\end{array}$ & $\begin{array}{c}\delta+1 \\
\mathcal{X}_{N_{R}}+\mathcal{X}_{X_{1}}\end{array}$ & $\begin{array}{c}\delta+1 \\
\mathcal{X}_{N_{R}}+\mathcal{X}_{X_{1}}+\mathcal{X}_{S}\end{array}$ & \\
\hline T4-3-I & $\begin{array}{l}U(1)_{\mathrm{L}} \\
U(1)_{\mathrm{PQ}}\end{array}$ & $-\left(\mathcal{X}_{N_{R}}^{-1}+\mathcal{X}_{S}\right)$ & $\begin{array}{c}\delta-1 \\
\mathcal{X}_{X_{4}}-\left(\mathcal{X}_{N_{R}}+\mathcal{X}_{S}\right)\end{array}$ & $\mathcal{X}_{X_{4}}{ }^{\delta}-\mathcal{X}_{S}$ & $\begin{array}{c}\delta \\
\mathcal{X}_{X_{4}}\end{array}$ \\
\hline
\end{tabular}

(iii) vanishing contributions of the tree-level realizations of $\mathcal{O}_{5}$ to the neutrino mass matrix, and (iv) existence of a remnant $Z_{N}$ symmetry after the PQ breaking (see next section). For completeness purposes, we display in the Appendix the sets of quantum numbers of the mediator fields for each model [21]. It turns out that for $\alpha=0,1$, or 2 (the values leading to either $Y=0$ singlet fermions, $Y= \pm 1$ doublet fermions, or $Y= \pm 1$ doublet scalars) one or two Dirac seesaw mechanisms arise, implying that the one-loop contribution is subdominant [the exceptional case is the T4-3-I-V model since all the mediators fields are $S U(2)_{L}$ triplets].

(1) T1 models: The six T1 models have four mediators, where the T1-1-A and T1-1-B models have three scalar mediators, T1-3-D and T1-3-E models have two scalars, whereas there is one single scalar mediator in T1-2-A and T1-2-B models. The T1 models differ on the number of scalar and fermion mediator fields or on the exchange of the $S$ and $H$. Note that models T1-1-A and T1-2-A share the same $\mathrm{L}$ and $\mathrm{PQ}$ charges, and this is the same for T1-1-B and T1-2-B models. This is because the charge assignment is independent of the $S U(2)_{L}$ transformation properties of the fields. Each T1 model has four sets of possible electroweak charges for the mediator fields, all of them featuring at least one of the mediators of the Dirac seesaw mechanisms when $\alpha=0,1$, or 2 (see Table IV).

(2) T3 models: There is one type of model with a T3 topology, which involves three mediators with two of them scalars. The T3 model has four sets of possible electroweak charges for the mediator fields, with at least one of the mediators of the Dirac seesaw mechanisms when $\alpha=0,1$, or 2 (see Table V).

(3) T4 models: The T4-3-I models have four mediators with two fermions, with the particular feature that $X_{1}$ is not running inside the loop. This, in turn, implies that all its quantum numbers are fixed. It transforms as a $S U(3)_{L}$ triplet with zero hypercharge, and the $\mathrm{L}$ and PQ charges are settled by the lepton doublet charges: $\mathrm{L}\left(X_{1}\right)=-1$ and $\mathcal{X}_{X_{1}}=-\mathcal{X}_{L}=-\left(\mathcal{X}_{N_{R}}+\mathcal{X}_{S}\right)$. On the other hand, the T4-3-I-V model does not contain any mediator field of the Dirac seesaw mechanisms, however, it requires the mixing between the neutral and charged leptons with the neutral and charged components of $X_{1}$, which induce charged lepton flavor violating processes at tree level.

In the next section, we will present the conditions on the charges of the mediator fields that must be fulfilled to guarantee, at the same time, the presence of a WIMP candidate in the spectrum and the absence of tree-level Dirac seesaw mechanisms.

\section{RADIATIVE NEUTRINO MASSES AND AXION-WIMP DARK MATTER}

It turns out that the same values for $\alpha$ that lead to treelevel Dirac seesaw mechanisms are the ones that allow us to have an electrically neutral and PQ charged particle in the spectrum, thus opening the possibility of having multicomponent DM scenarios comprising such particle (a WIMP) and the axion. Concretely, the existence of a neutral particle in the spectrum is guaranteed by setting $Q=T_{3}+Y / 2=0$, such that $Y=-2 T_{3}$ for at least one of the mediators. This implies that $\alpha$ can only take the values 0 , 1, or 2 [depending upon the $S U(2)_{L}$ representation], which are precisely the same values that lead to the treelevel realizations of $\mathcal{O}_{5}$. Thus, the presence of a WIMP candidate in the models automatically implies that the oneloop contribution is the main contribution to the neutrino masses. 
Regarding the stability of the WIMP particle, all the mediator fields (except $X_{1}$ in T4 models ${ }^{5}$ ) can be made part of a dark (WIMP) sector by demanding that a residual $Z_{N}$ symmetry is generated after the $U(1)_{\mathrm{PQ}}$ breaking, in the same line of the scotogenic models. This is achieved when $\mathcal{X}_{S}$ is an even integer $N$ (to preserve the periodicity of the axion potential) and the PQ charges of the loop mediators are $\mathcal{X}_{X_{i}} \neq 0 \bmod \left(\mathcal{X}_{S}\right)$.

On the other hand, the conditions over the PQ charges of the mediator fields that avoid tree-level Dirac seesaw mechanisms (and hence guarantee a WIMP particle in the spectrum) lead to PQ charges $\mathcal{X}_{X_{i}}$ of the form (see Table I)

$$
\mathcal{X}_{X_{4}}, \mathcal{X}_{X_{4}} \pm \mathcal{X}_{S}, \mathcal{X}_{X_{4}} \pm \mathcal{X}_{N_{R}}, \quad \text { or } \quad \mathcal{X}_{X_{4}} \pm \mathcal{X}_{N_{R}} \pm \mathcal{X}_{S}
$$

(for T1 and T4 models),

$$
\mathcal{X}_{X_{1}}, \mathcal{X}_{X_{1}} \pm \mathcal{X}_{N_{R}}, \quad \text { or } \quad \mathcal{X}_{X_{1}} \pm \mathcal{X}_{N_{R}} \pm \mathcal{X}_{S}
$$

(for T3 models).

Consequently, in order to ensure a nontrivial charge for these fields under the remnant $Z_{N}\left(N=\mathcal{X}_{S}\right)$, the $\mathcal{X}_{X_{4}}$ charge $\left(\mathcal{X}_{X_{1}}\right.$ for T3-1-A model) must be an integer nonmultiple of $\mathcal{X}_{S}$ as long as $N_{R}$ transforms trivially, $\mathcal{X}_{N_{R}}=0 \bmod \left(\mathcal{X}_{S}\right)$ (this the simplest choice, however, see Sec. IV for other possible $Z_{N}$ assignment). Note further that if $\mathcal{X}_{N_{R}} \neq 0$ and $2 \mathcal{X}_{N_{R}} \neq \mathcal{X}_{S}$ the L conservation would not be mandatory since the Diracness of neutrinos would be guarantee for the $Z_{N}$ properties of the lepton doublet, $L \not \omega^{N / 2}$ (see Ref. [66] for details and Sec. IV for an example).

To illustrate a specific charge assignment, let us assume $\mathcal{X}_{S}=2$ and $\mathcal{X}_{N_{R}}=0$, which entails that the PQ charge of the lepton doublet and singlet are both two (this is because of the Yukawa terms $\bar{L} \tilde{H} N_{R} S$ and $\bar{L} H \ell_{R}$ ). Thus, if all the mediators running inside the loop have odd PQ charges, then a remnant $Z_{2}$ symmetry would appear in such a way that they are $Z_{2}$ odd, while the rest of the model particles are $Z_{2}$ even.

The stability of electrically neutral particles naturally leads to WIMP scenarios. Among the possible scenarios that may arise (see Tables IV-VI) we highlight the ultraviolet fermionic realizations of the Higgs portal [67] — the singlet (via the axion interactions), singlet-doublet, and doublet-triplet Dirac DM [68-71] — and the renormalizable scalar DM models: the singlet scalar DM model [72-74], inert doublet model (IDM) [75,76], and inert triplet model [77-79], along with the interplays singlet doublet and doublet triplet [80-84].

Regarding the heavy quark $D$, since the SM quarks and $H$ are not charged under the PQ symmetry, $D_{R}$ cannot

\footnotetext{
${ }^{5}$ It is worth mentioning that in the T4 models the $X_{1}$ field is not running inside the loop, which makes it different from the other mediator fields in what concerns DM.
}

couple to the SM quark doublet through $\overline{q_{L}} H D_{R}$ as long as $\mathcal{X}_{D_{R}} \neq 0$. However, it may interact with the quark singlet via the $\bar{D}_{L} d_{R} S$ term, which entails that $\mathcal{X}_{D_{L}} \neq \mathcal{X}_{S}$ to avoid the constraints due to the mixing the SM quarks. With respect to the interactions with the dark sector, if there exists a $Y=0$ singlet scalar $\varphi$ or a second Higgs doublet $\mathrm{H}_{2}$ in the spectrum, then the terms $\overline{D_{L}} d_{R} \varphi$ and $\overline{q_{L}} H_{2} D_{R}$ would appear. The first term would require that $\mathcal{X}_{D_{L}}=\mathcal{X}_{\varphi}$, while the second one $\mathcal{X}_{D_{R}}=-\mathcal{X}_{H_{2}}$, so $D$ must be also part of the dark sector in order for such terms be allowed. It follows that $D$ can only decay into dark scalars ( $\varphi$ and/or $\mathrm{H}_{2}$ ) and SM quarks, leading to supersymmetrylike signals, such as jets plus missing energy at the LHC if $D$ is not heavy enough (see, e.g., Ref. [34] for an explicit analysis). In the opposite case, the Lagrangian would be invariant under the exchange $\left(D_{L}, D_{R}\right) \rightarrow\left(-D_{L},-D_{R}\right)$, making it a stable colored heavy quark, thus bringing some cosmological problems [85]. Therefore, the instability of the heavy quark $D$ imposes additional constraints over the viable models since it demands the existence of either a $Y=0$ singlet scalar or an extra Higgs doublet in the dark sector. From Tables IV-VI, we obtain that the following models do not contain such fields: T1-2-A-IV, T1-3-D-IV, and T4-3-I-V.

On the other hand, since the PQ mechanism is at work, suitable parameters for the QCD axion can be considered in order to have the axion as a second DM candidate. In the framework of a broad class of inflationary scenarios, QCD axions may be nonthermally produced in the early Universe through the vacuum-realignment mechanism and surviving as cold DM matter. The axion contribution to energy density depends on the order in which cosmological events take place, especially whether the breaking of the PQ symmetry occurred before or after inflation. For the case of reheating temperatures lower than $f_{a}$, the axion relic density due to the misalignment population is fixed by the initial field displacement, the so-called misalignment angle $\theta_{a}$, and $f_{a}[86,87]$,

$$
\Omega_{a} h^{2} \approx 0.18 \theta_{a}^{2}\left(\frac{f_{a}}{10^{12} \mathrm{GeV}}\right)^{1.19} .
$$

Thus, the allowed window of axion parameters is

$$
\begin{gathered}
10^{9} \lesssim f_{a} \lesssim \theta_{a}^{-2} 10^{12} \mathrm{GeV} \\
6 \theta_{a}^{2} \mu \mathrm{eV} \lesssim m_{a} \lesssim 6 \mathrm{meV}
\end{gathered}
$$

where $m_{a} \approx 6 \mu \mathrm{eV}\left(10^{12} \mathrm{GeV} / f_{a}\right)[59,60]$ (see Refs. [88,89] for a detailed study of the window for preferred axion models and Refs. [90-94] for the current status of experimental searches). For this case, the axion population from the decay of topological defects such as string axions and domain walls is diluted by inflation. 
It follows that, in this framework, the DM of the Universe comprises the WIMP and the axion, which behave as two completely independent DM particles, without affecting the standard relic density calculations and the corresponding experimental bounds [31-36,95]. Therefore, at the electroweak scale, the models presented in this work are up to some extent similar to the renormalizable WIMP DM models mentioned above, with the main difference being that the DM is complemented by axions. And since there exist two contributions to the total DM relic abundance, we expect that all the constraints over such WIMP scenarios would be substantially weakened, thus resulting in larger portions of the parameter space that are still allowed within those scenarios (see Refs. [33,34,36,95] for specific scenarios). In addition to this, since the WIMP may couple to the right-handed neutrino through Yukawa interactions, further WIMP annihilation channels may appear, thus changing drastically the expected DM phenomenology, with the bonus that they are rather unconstrained because such interactions do not induce lepton flavor violation processes.

\section{A CASE STUDY}

In this section we study a specific model resulting from the analysis made in previous sections, the T3-1-A-I model with $\alpha=0$ and a residual $Z_{2}$ symmetry. Thus, the model contains the following fields: an $S U(2)_{L}$ doublet scalar $X_{3}$ and two SM singlets, one being a Dirac fermion $X_{1}$, while the other one is a complex scalar $X_{2}$. We add a second singlet fermion in order to obtain two massive neutrinos. In what follows we rename these fields as $X_{1} \rightarrow \psi^{c}, X_{2} \rightarrow \varphi^{*}$, and $X_{3} \rightarrow \tilde{H}_{2}$, with $\left.\varphi=\left(\varphi_{R}+i \varphi_{I}\right) / \sqrt{2}, H_{2}=\left(H^{+},\left(H^{0}+i A^{0}\right) / \sqrt{2}\right)\right)^{T}$, and $\tilde{H}_{2}=i \sigma_{2} H_{2}^{*}$. Moreover, $H_{1}$ will denote the SM Higgs doublet. The $U(1)_{\mathrm{PQ}}$ and $U(1)_{\mathrm{L}}$ charges are given in Table II.

The more general Lagrangian invariant under $\mathcal{G}_{\mathrm{SM}} \times$ $U(1)_{\mathrm{L}} \times U(1)_{\mathrm{PQ}}$ symmetry comprises the interaction terms $-\mathcal{L} \supset \mathcal{V}_{H_{1}, S}+\mathcal{V}_{\varphi}+\mathcal{V}_{H_{2}}+\mathcal{V}_{1}+\mathcal{L}_{1}$, where

$\mathcal{V}_{H_{1}, S}=-\mu_{1}^{2}\left|H_{1}\right|^{2}+\lambda_{1}\left|H_{1}\right|^{4}+\mu_{S}^{2}|S|^{2}+\lambda_{S}|S|^{4}+\lambda_{6}\left|H_{1}\right|^{2}|S|^{2}$,

TABLE II. L and PQ charges for model T3-1-A-I with $\alpha=0$ and $\delta=-1, \mathcal{X}_{S}=2, \mathcal{X}_{\psi}=1$, and $\mathcal{X}_{N_{R}}=0$. The charges under the remnant $Z_{2}$ symmetry are also shown.

\begin{tabular}{lccccccccc}
\hline \hline & $L$ & $\ell_{R}$ & $N_{R}$ & $S$ & $\psi$ & $\varphi$ & $H_{2}$ & $D_{L}$ & $D_{R}$ \\
\hline$U(1)_{\mathrm{L}}$ & 1 & 1 & 1 & 0 & 1 & 0 & 0 & 0 & 0 \\
$U(1)_{\mathrm{PQ}}$ & 2 & 2 & 0 & 2 & 3 & 3 & 1 & 1 & -1 \\
$Z_{2}$ & + & + & + & + & - & - & - & - & - \\
\hline \hline
\end{tabular}

$$
\begin{gathered}
\mathcal{V}_{\varphi}=\mu_{\varphi}^{2}|\varphi|^{2}+\lambda_{\varphi}|\varphi|^{4}+\lambda_{7}\left|H_{1}\right|^{2}|\varphi|^{2}+\lambda_{8}|\varphi|^{2}|S|^{2}, \\
\mathcal{V}_{H_{2}}=\mu_{2}^{2}\left|H_{2}\right|^{2}+\lambda_{2}\left|H_{2}\right|^{4}+\lambda_{3}\left|H_{1}\right|^{2}\left|H_{2}\right|^{2} \\
+\lambda_{4}\left|H_{1}^{\dagger} H_{2}\right|^{2}+\lambda_{9}\left|H_{2}\right|^{2}|S|^{2}, \\
\mathcal{V}_{1}=\lambda_{10}\left|H_{2}\right|^{2}|\varphi|^{2}+\lambda_{11}\left[\varphi^{*} S \tilde{H}_{2}^{\dagger} \tilde{H}_{1}+\text { H.c. }\right], \\
\mathcal{L}_{1}=y_{i \beta} \bar{\psi}_{i} \tilde{H}_{2}^{\dagger} L_{\beta}+h_{\beta i} \varphi^{*} \overline{N_{R \beta}} \psi_{i}+y_{Q} S \bar{D}_{L} D_{R} \\
+f_{\beta} \bar{q}_{L \beta} H_{2} D_{R}+\text { H.c. }
\end{gathered}
$$

Here some comments are in order. The $\lambda_{6}$ term mixes the real component of the axion with the Higgs field, so we can neglect it without lost of generality. $\lambda_{11}$ is responsible for the mixing between the neutral components of $\varphi$ and $H_{2}$ that leads to Dirac neutrino masses (notice that $\lambda_{11}$ plays, to some extent, the role of the $\lambda_{5}$ term in the scotogenic model [96]). Since the SM quarks are not charged under the PQ symmetry, $D$ does not couple to SM particles through $\overline{D_{L}} d_{R} \varphi$, but does through $\overline{q_{L}} H_{2} D_{R}$. Note that, under the $Z_{2}$ remnant symmetry, ${ }^{6}$ the fields running in the loop of diagram T3-1-A in Fig. 1 are odd. Therefore, the lightest of them can be considered as the DM candidate (if it is neutral). This model at low energies, i.e., without the axion, is identical to the one presented in Ref. [10], since instead of considering an ultraviolet completion realization, it introduced a soft breaking term that allows the mixing between the neutral components of the singlet and doublet (in a similar way as $\lambda_{11}$ does).

Let us consider the limit $\lambda_{6} \rightarrow 0$, in order to avoid the mixing between the scalar $S$ and the SM Higgs boson, and the limit $\lambda_{8,9} \rightarrow 0$ with the aim to avoid a large fine-tuning in the masses of $\varphi$ and $H^{0}$. In the basis $\left(H^{0}, \varphi_{R}\right)^{T}$ and $\left(A^{0}, \varphi_{I}\right)^{T}$, the mass matrices for the $Z_{2}$-odd neutral scalars are given by

$$
\begin{aligned}
\mathcal{M}_{R, I} & =\left(\begin{array}{cc}
\mu_{2}^{2}+\frac{1}{2}\left(\lambda_{3}+\lambda_{4}\right) v^{2} & \frac{1}{2} \lambda_{11} v_{S} v \\
\frac{1}{2} \lambda_{11} v_{S} v & \mu_{\varphi}^{2}+\frac{1}{2} \lambda_{7} v^{2}
\end{array}\right), \\
\sin \left(2 \theta_{R, I}\right) & =\frac{\lambda_{11} v v_{S}}{m_{S_{R, I 2}^{2}}^{2}-m_{S_{R, I 1}}^{2}},
\end{aligned}
$$

where $m_{S_{R j}}\left(m_{S_{I j}}\right)$ are the two mass eigenvalues of $\mathcal{M}_{R}$ $\left(\mathcal{M}_{I}\right)$. The mass of the charged scalar $H^{+}$is given by $m_{H^{+}}^{2}=\mu_{2}^{2}+\frac{1}{2} \lambda_{3} v^{2}$, as in the IDM.

Neutrino masses are generated at one loop through the Feynman diagram, corresponding to the T3-1-A model in Fig. 1. The effective mass matrix is given by

\footnotetext{
${ }^{6}$ In fact, a larger dark U(1) symmetry is obtained, which contains the remnant $Z_{2}$ as a subgroup.
} 


$$
\begin{aligned}
\left(M_{\nu}\right)_{\beta \beta^{\prime}}= & \frac{1}{64 \pi^{2}} \frac{\lambda_{11} v_{S} v}{m_{S_{R 2}}^{2}-m_{S_{R 1}}^{2}} \\
& \times \sum_{i} h_{\beta i} y_{i \beta^{\prime}} m_{\psi_{i}}\left[F\left(\frac{m_{S_{R 2}}^{2}}{m_{\psi_{i}}^{2}}\right)-F\left(\frac{m_{S_{R 1}}^{2}}{m_{\psi_{i}}^{2}}\right)\right] \\
& +(R \rightarrow I),
\end{aligned}
$$

where $F(x)=x \ln (x) /(x-1)$, and $R \rightarrow I$ means the exchange of the $C P$-even eigenstates $\left(S_{R j}\right)$ with the corresponding $C P$-odd eigenstates $\left(S_{I j}\right)$. It follows that the neutrino masses are suppressed by several factors: the loop factor, the Yukawa couplings, the mass of the heaviest loop mediator, and $\lambda_{11}$. It turns out that the resulting suppression is enough to counteract the contribution coming from $v_{S}$ [see Eq. (17)]. A simple numerical estimate for the effective mass matrix can be calculated considering the $\operatorname{limit}^{7} \lambda_{3,4,7} \rightarrow 0, \mu_{2}^{2} \rightarrow \mu_{\varphi}^{2}$, and $\mu_{\varphi}^{2} \gg$ $\lambda_{11} v_{S} v$. In this case,

$$
\begin{aligned}
\left(M_{\nu}\right)_{\beta \beta^{\prime}} \approx & \frac{\lambda_{11} v_{S} v}{32 \pi^{2}} \sum_{i} h_{\beta i} y_{i \beta^{\prime}} \frac{m_{\psi_{i}}}{\mu_{\varphi}^{2}-m_{\psi_{i}}^{2}} \\
& \times\left[1-\frac{m_{\psi_{i}}^{2}}{\mu_{\varphi}^{2}-m_{\psi_{i}}^{2}} \log \left(\frac{\mu_{\varphi}^{2}}{m_{\psi_{i}}^{2}}\right)\right] .
\end{aligned}
$$

If $\mu_{\varphi}^{2} \gg m_{\psi}^{2}$, that is, considering fermion DM, it reduces to

$$
\begin{aligned}
\left(M_{\nu}\right)_{\beta \beta^{\prime}} \approx & \frac{\lambda_{11} v_{S} v}{32 \pi^{2}} \sum_{i} h_{\beta i} y_{i \beta^{\prime}} \frac{m_{\psi_{i}}}{\mu_{\varphi}^{2}} \\
\sim & 0.05 \mathrm{eV} \times\left(\frac{h_{\beta i} y_{i \beta^{\prime}}}{10^{-4}}\right)\left(\frac{\lambda_{11}}{10^{-10}}\right)\left(\frac{m_{\psi_{i}}}{10^{2} \mathrm{GeV}}\right) \\
& \times\left(\frac{5 \times 10^{3} \mathrm{GeV}}{\mu_{\varphi}}\right)^{2}\left(\frac{v_{S}}{10^{9} \mathrm{GeV}}\right) .
\end{aligned}
$$

The linear dependence of the neutrino masses with $v_{S}$ arises because the loop mediators are not lying at the PQ scale, a feature that is also shared with other radiative neutrino mass models, e.g., [48]. Note that the smallness of $\lambda_{11}$ is natural in the ' $t$ Hooft sense [97], since $\lambda_{11}=0$ leads to an extra symmetry (the charge of $S$ is no longer connected with the charges of the other particles). Furthermore, the smallness of $\lambda_{11}$ is a necessary condition in order to have the loop scalar mediators at or below the TeV mass scale. This is also in consonance with the requirement of demanding a tiny value for the scalar coupling between the Higgs boson $H_{1}$ and the axion field $S$ (these conditions are unavoidable in axion models as long as light loop mediators are required [33]).

On the other hand, the Yukawa interactions in Eq. (13) also lead to lepton flavor violation processes at one loop

\footnotetext{
${ }^{7}$ The limits $\lambda_{3,4,7} \rightarrow 0$ are motivated by the naturalness of the scalar potential since they avoid the imposition of large cancellations among the scalar potential parameters.
}

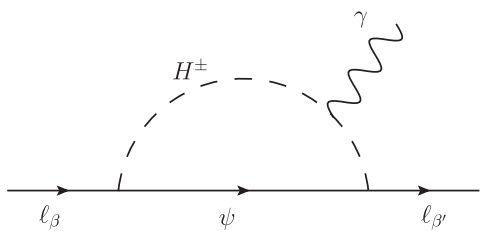

FIG. 2. Feynman diagram for the process $\ell_{\beta} \rightarrow \ell_{\beta^{\prime}} \gamma$.

mediated by the charged scalar $H^{ \pm}$and $\psi_{i}$ (see Fig. 2). The decay rate for this process is given by

$$
\begin{aligned}
\Gamma\left(\ell_{\beta} \rightarrow \ell_{\beta^{\prime}} \gamma\right)= & \frac{e^{2} m_{\beta}^{5}}{16 \pi} \frac{1}{16^{2} \pi^{4} m_{H^{ \pm}}^{4}} \\
& \times \sum_{i}\left|y_{i \beta} y_{i \beta^{\prime}}^{*}\right|^{2}\left(\frac{2 t_{i}^{2}+5 t_{i}-1}{12\left(t_{i}-1\right)^{3}}-\frac{t_{i}^{2} \log t_{i}}{2\left(t_{i}-1\right)^{4}}\right)^{2},
\end{aligned}
$$

where $t_{i} \equiv m_{\psi_{i}}^{2} / m_{H^{ \pm}}^{2}$. Considering the limit of the heavy scalar, $m_{H^{ \pm}} \gg m_{\psi_{i}}$, it follows that

$$
\left|y_{i \mu} y_{i e}^{*}\left(\frac{5 \times 10^{3} \mathrm{GeV}}{m_{H^{ \pm}}}\right)^{2}\right| \lesssim 0.1
$$

where the bound $\operatorname{BR}(\mu \rightarrow e \gamma)<5.7 \times 10^{-13}$ [98] has been used.

Regarding WIMP DM, this model features either singlet fermion or singlet-doublet scalar candidates. ${ }^{8}$ Such a WIMP-like setup is similar to the model studied in Ref. [10] with the difference that in the present case the DM is additionally composed by axions. Therefore, all the constraints that may exist on the model with only WIMPs would be substantially weakened. Indeed, when the WIMP particle is mainly doublet (an IDM-like scenario) most of the intermediate DM mass range can be free of DM constraints [33,34] (see Ref. [36] for the case of singlet scalar DM). In the case of fermion DM, in order to account for the total relic abundance, the $\bar{\psi} \psi$ annihilation must be driven by $h_{\beta i}$ Yukawa terms, since in this case there are not constraints coming from lepton flavor violating processes, so they can be large enough [10]. In contrast, $\bar{\psi} \psi$ annihilation via $y_{i \beta}$ Yukawa terms is not a viable option without some detailed fine-tuning of parameters. All in all, this shows the impact of considering mixed axion-WIMP DM scenarios.

Finally, it is worth mentioning that, with a suitable PQ charge assignment (such as the one displayed in Table III) and without imposing lepton number conservation, it is possible to obtain a consistent model where the Diracness of neutrinos is guaranteed. In this case, a remnant $Z_{4}$

\footnotetext{
${ }^{8}$ A detailed DM phenomenological study of this model will be done elsewhere.
} 
TABLE III. Alternative PQ charges for model T3-1-A-I that guarantee the Diracness of neutrinos without further assuming the lepton number symmetry. The charges under the remnant $Z_{4}$ symmetry are also shown, where $\omega^{4}=1$.

\begin{tabular}{lccccccccc}
\hline \hline & $L$ & $\ell_{R}$ & $N_{R}$ & $S$ & $\psi$ & $\varphi$ & $H_{2}$ & $D_{L}$ & $D_{R}$ \\
\hline$U(1)_{\mathrm{PQ}}$ & 8 & 8 & 4 & 4 & 7 & 3 & -1 & 5 & 1 \\
$Z_{4}$ & 1 & 1 & 1 & 1 & $\omega^{3}$ & $\omega^{3}$ & $\omega^{3}$ & $\omega$ & $\omega$ \\
\hline \hline
\end{tabular}

symmetry is obtained from the PQ symmetry breaking, which is responsible for the stability the WIMP candidate.

\section{SUMMARY}

In this work we have studied the one-loop realizations of the $d=5$ operator $\bar{L} \tilde{H} N_{R} S$ that leads to Dirac neutrino masses, with $S$ being a singlet scalar field that hosts the QCD axion. As usual, the axion arises from the breaking of the Peccei-Quinn symmetry, which in our setup we used to not only solve the strong $C P$ problem, but also to forbid the operator $\bar{L} \tilde{H} N_{R}$ (which generates Dirac neutrino masses at tree level) and the tree-level realizations of $\bar{L} \tilde{H} N_{R} S$. Thus, the neutrino masses are directly correlated to the axion mass (via the PQ symmetry breaking scale $v_{s}$ ) and their smallness is due to the radiative character besides the mass suppression of the loop mediators. Furthermore, the PQ symmetry breaking leaves a residual $Z_{N}$ symmetry that allow us to guarantee the stability of the lightest of the mediators in the one-loop neutrino mass diagrams (as happens in the scotogenic models), thus leading naturally to multicomponent DM scenarios with axions and WIMPs. We have illustrated our proposal by considering a specific model, where simple numerical estimates allow us to show the effectiveness of the scheme regarding neutrino masses, $\mathrm{DM}$, and lepton flavor violating processes.

\section{ACKNOWLEDGMENTS}

We are thankful to Diego Restrepo for enlightening discussions. Work supported by Sostenibilidad-UdeA and the UdeA/CODI Grant No. 2017-16286 and by COLCIENCIAS through the Grants No. 111565842691 and No. 111577657253. C. D. R. C. acknowledges the financial support given by the Departamento Administrativo de Ciencia, Tecnología e InnovaciónCOLCIENCIAS (doctoral scholarship 727-2015). O.Z. acknowledges the ICTP Simons associates program and the kind hospitality of the Abdus Salam ICTP where part of this work was done.

\section{APPENDIX: MEDIATOR FIELDS QUANTUM NUMBER SETS}

Below we display the sets of quantum numbers of the mediator fields for each model [21].
TABLE IV. Possible quantum numbers for the mediators in the T1 topologies.

(a)

\begin{tabular}{lcccccc}
\hline Model & Solution & $X_{1}^{F}$ & $X_{2}^{S}$ & $X_{3}^{S}$ & $X_{4}^{S}$ & $\alpha$ \\
\hline & I & $1_{\alpha}$ & $1_{\alpha}$ & $1_{\alpha}$ & $2_{\alpha-1}$ & 0,2 \\
T1-1-A & II & $2_{\alpha}$ & $2_{\alpha}$ & $2_{\alpha}$ & $1_{\alpha-1}$ & \pm 1 \\
& III & $2_{\alpha}$ & $2_{\alpha}$ & $2_{\alpha}$ & $3_{\alpha-1}$ & \pm 1 \\
& IV & $3_{\alpha}$ & $3_{\alpha}$ & $3_{\alpha}$ & $2_{\alpha-1}$ & 0,2 \\
& I & $1_{\alpha}$ & $1_{\alpha}$ & $2_{\alpha-1}$ & $2_{\alpha-1}$ & 0,2 \\
T1-1-B & II & $2_{\alpha}$ & $2_{\alpha}$ & $1_{\alpha-1}$ & $1_{\alpha-1}$ & \pm 1 \\
& III & $2_{\alpha}$ & $2_{\alpha}$ & $3_{\alpha-1}$ & $3_{\alpha-1}$ & \pm 1 \\
& IV & $3_{\alpha}$ & $3_{\alpha}$ & $2_{\alpha-1}$ & $2_{\alpha-1}$ & 0,2 \\
\hline \hline
\end{tabular}

(b)

\begin{tabular}{ccccccc}
\hline Model & Solution & $X_{1}^{S}$ & $X_{2}^{F}$ & $X_{3}^{F}$ & $X_{4}^{F}$ & $\alpha$ \\
\hline \multirow{4}{*}{ T1-2-A } & I & $1_{\alpha}$ & $1_{\alpha}$ & $1_{\alpha}$ & $2_{\alpha-1}$ & 0,2 \\
& II & $2_{\alpha}$ & $2_{\alpha}$ & $2_{\alpha}$ & $1_{\alpha-1}$ & \pm 1 \\
& II & $2_{\alpha}$ & $2_{\alpha}$ & $2_{\alpha}$ & $3_{\alpha-1}$ & \pm 1 \\
& IV & $3_{\alpha}$ & $3_{\alpha}$ & $3_{\alpha}$ & $2_{\alpha-1}$ & 0,2 \\
T1-2-B & I & $1_{\alpha}$ & $1_{\alpha}$ & $2_{\alpha-1}$ & $2_{\alpha-1}$ & 0,2 \\
& II & $2_{\alpha}$ & $2_{\alpha}$ & $1_{\alpha-1}$ & $1_{\alpha-1}$ & \pm 1 \\
& III & $2_{\alpha}$ & $2_{\alpha}$ & $3_{\alpha-1}$ & $3_{\alpha-1}$ & \pm 1 \\
& IV & $3_{\alpha}$ & $3_{\alpha}$ & $2_{\alpha-1}$ & $2_{\alpha-1}$ & 0,2
\end{tabular}

(c)

\begin{tabular}{ccccccc}
\hline Model & Solution & $X_{1}^{F}$ & $X_{2}^{S}$ & $X_{3}^{S}$ & $X_{4}^{F}$ & $\alpha$ \\
\hline \multirow{4}{*}{ T1-3-D } & I & $1_{\alpha}$ & $1_{\alpha}$ & $1_{\alpha}$ & $2_{\alpha+1}$ & 0 \\
& II & $2_{\alpha}$ & $2_{\alpha}$ & $2_{\alpha}$ & $1_{\alpha+1}$ & \pm 1 \\
& III & $2_{\alpha}$ & $2_{\alpha}$ & $2_{\alpha}$ & $3_{\alpha+1}$ & \pm 1 \\
& IV & $3_{\alpha}$ & $3_{\alpha}$ & $3_{\alpha}$ & $2_{\alpha+1}$ & 0 \\
T1-3-E & I & $1_{\alpha}$ & $1_{\alpha}$ & $2_{\alpha-1}$ & $1_{\alpha}$ & 0,2 \\
& II & $2_{\alpha}$ & $2_{\alpha}$ & $1_{\alpha-1}$ & $2_{\alpha}$ & \pm 1 \\
& III & $2_{\alpha}$ & $2_{\alpha}$ & $3_{\alpha-1}$ & $2_{\alpha}$ & \pm 1 \\
& IV & $3_{\alpha}$ & $3_{\alpha}$ & $2_{\alpha-1}$ & $3_{\alpha}$ & 0,2 \\
\hline \hline
\end{tabular}

TABLE V. Possible quantum numbers for the mediators in the T3 topology.

\begin{tabular}{cccccc}
\hline \hline Model & Solution & $X_{1}^{F}$ & $X_{2}^{S}$ & $X_{3}^{S}$ & $\alpha$ \\
\hline \multirow{3}{*}{ T3-1-A } & I & $1_{\alpha}$ & $1_{\alpha}$ & $2_{\alpha-1}$ & 0,2 \\
& II & $2_{\alpha}$ & $2_{\alpha}$ & $1_{\alpha-1}$ & \pm 1 \\
& III & $2_{\alpha}$ & $2_{\alpha}$ & $3_{\alpha-1}$ & \pm 1 \\
& IV & $3_{\alpha}$ & $3_{\alpha}$ & $2_{\alpha-1}$ & 0,2 \\
\hline \hline
\end{tabular}

TABLE VI. Possible quantum numbers for the mediators in the T4 topology.

\begin{tabular}{lcccccc}
\hline \hline Model & Solution & $X_{1}^{F}$ & $X_{2}^{F}$ & $X_{3}^{S}$ & $X_{4}^{S}$ & $\alpha$ \\
\hline \multirow{2}{*}{ T4-3-I } & IV & $3_{0}$ & $2_{\alpha}$ & $2_{\alpha}$ & $2_{\alpha}$ & \pm 1 \\
& $\mathrm{~V}$ & $3_{0}$ & $3_{\alpha}$ & $3_{\alpha}$ & $3_{\alpha}$ & \\
\hline \hline
\end{tabular}


[1] M. Tanabashi et al. (Particle Data Group), Review of particle physics, Phys. Rev. D 98, 030001 (2018).

[2] P. F. de Salas, D. V. Forero, C. A. Ternes, M. Tortola, and J. W. F. Valle, Status of neutrino oscillations 2018: $3 \sigma$ hint for normal mass ordering and improved $C P$ sensitivity, Phys. Lett. B 782, 633 (2018).

[3] A. Gando et al. (KamLAND-Zen Collaboration), Search for Majorana Neutrinos near the Inverted Mass Hierarchy Region with KamLAND-Zen, Phys. Rev. Lett. 117, 082503 (2016); Erratum, Phys. Rev. Lett. 117, 109903(E) (2016).

[4] M. Agostini et al. (GERDA Collaboration), Improved Limit on Neutrinoless Double- $\beta$ Decay of ${ }^{76} \mathrm{Ge}$ from GERDA Phase II, Phys. Rev. Lett. 120, 132503 (2018).

[5] C. E. Aalseth et al. (Majorana Collaboration), Search for Neutrinoless Double- $\beta$ Decay in ${ }^{76} \mathrm{Ge}$ with the Majorana Demonstrator, Phys. Rev. Lett. 120, 132502 (2018).

[6] C. Alduino et al. (CUORE Collaboration), First Results from CUORE: A Search for Lepton Number Violation via $0 \nu \beta \beta$ Decay of ${ }^{130}$ Te, Phys. Rev. Lett. 120, 132501 (2018).

[7] J. B. Albert et al. (EXO Collaboration), Search for Neutrinoless Double-Beta Decay with the Upgraded EXO-200 Detector, Phys. Rev. Lett. 120, 072701 (2018).

[8] R. Arnold et al. (NEMO-3 Collaboration), Measurement of the $2 \nu \beta \beta$ decay half-life and search for the $0 \nu \beta \beta$ decay of ${ }^{116} \mathrm{Cd}$ with the NEMO-3 detector, Phys. Rev. D 95, 012007 (2017).

[9] G. Steigman and M. S. Turner, Cosmological constraints on the properties of weakly interacting massive particles, Nucl. Phys. B253, 375 (1985).

[10] Y. Farzan and E. Ma, Dirac neutrino mass generation from dark matter, Phys. Rev. D 86, 033007 (2012).

[11] E. Ma and R. Srivastava, Dirac or inverse seesaw neutrino masses with $B-L$ gauge symmetry and $S_{3}$ flavor symmetry, Phys. Lett. B 741, 217 (2015).

[12] E. Ma and R. Srivastava, Dirac or inverse seesaw neutrino masses from gauged $B--L$ symmetry, Mod. Phys. Lett. A 30, 1530020 (2015).

[13] E. Ma, N. Pollard, R. Srivastava, and M. Zakeri, Gauge $B-L$ model with residual $Z_{3}$ symmetry, Phys. Lett. B 750, 135 (2015).

[14] E. Ma and O. Popov, Pathways to naturally small Dirac neutrino masses, Phys. Lett. B 764, 142 (2017).

[15] W. Wang and Z.-L. Han, Naturally small Dirac neutrino mass with intermediate $S U(2)_{L}$ multiplet fields, J. High Energy Phys. 04 (2017) 166.

[16] C. Bonilla and J. W. F. Valle, Naturally light neutrinos in Diracon model, Phys. Lett. B 762, 162 (2016).

[17] S. C. Chuliá, E. Ma, R. Srivastava, and J. W. F. Valle, Dirac neutrinos and dark matter stability from lepton quarticity, Phys. Lett. B 767, 209 (2017).

[18] D. Borah and A. Dasgupta, Common origin of neutrino mass, dark matter and Dirac leptogenesis, J. Cosmol. Astropart. Phys. 12 (2016) 034.

[19] W. Wang, R. Wang, Z.-L. Han, and J.-Z. Han, The $B-L$ scotogenic models for Dirac neutrino masses, Eur. Phys. J. C 77, 889 (2017).

[20] C.-Y. Yao and G.-J. Ding, Systematic study of one-loop Dirac neutrino masses and viable dark matter candidates, Phys. Rev. D 96, 095004 (2017).
[21] C.-Y. Yao and G.-J. Ding, Systematic analysis of Dirac neutrino masses from a dimension five operator, Phys. Rev. D 97, 095042 (2018).

[22] E. Ma and U. Sarkar, Radiative left-right Dirac neutrino mass, Phys. Lett. B 776, 54 (2018).

[23] M. Reig, D. Restrepo, J. W. F. Valle, and O. Zapata, Boundstate dark matter and Dirac neutrino masses, Phys. Rev. D 97, 115032 (2018).

[24] S. C. Chuliá, R. Srivastava, and J. W. F. Valle, Seesaw roadmap to neutrino mass and dark matter, Phys. Lett. B 781, 122 (2018).

[25] H. Baer, K.-Y. Choi, J. E. Kim, and L. Roszkowski, Dark matter production in the early Universe: Beyond the thermal WIMP paradigm, Phys. Rep. 555, 1 (2015).

[26] G. Bertone, The moment of truth for WIMP dark matter, Nature (London) 468, 389 (2010).

[27] M. Escudero, A. Berlin, D. Hooper, and M.-X. Lin, Toward (finally!) ruling out $\mathrm{Z}$ and Higgs mediated dark matter models, J. Cosmol. Astropart. Phys. 12 (2016) 029.

[28] G. Arcadi, M. Dutra, P. Ghosh, M. Lindner, Y. Mambrini, M. Pierre, S. Profumo, and F. S. Queiroz, The waning of the WIMP? A review of models, searches, and constraints, Eur. Phys. J. C 78, 203 (2018).

[29] K. M. Zurek, Multi-component dark matter, Phys. Rev. D 79, 115002 (2009).

[30] S. Profumo, K. Sigurdson, and L. Ubaldi, Can we discover multi-component WIMP dark matter?, J. Cosmol. Astropart. Phys. 12 (2009) 016.

[31] H. Baer, A. Lessa, S. Rajagopalan, and W. Sreethawong, Mixed axion/neutralino cold dark matter in supersymmetric models, J. Cosmol. Astropart. Phys. 06 (2011) 031.

[32] K. J. Bae, H. Baer, and E. J. Chun, Mixed axion/neutralino dark matter in the SUSY DFSZ axion model, J. Cosmol. Astropart. Phys. 12 (2013) 028.

[33] B. Dasgupta, E. Ma, and K. Tsumura, Weakly interacting massive particle dark matter and radiative neutrino mass from Peccei-Quinn symmetry, Phys. Rev. D 89, 041702 (2014).

[34] A. Alves, D. A. Camargo, A. G. Dias, R. Longas, C. C. Nishi, and F. S. Queiroz, Collider and dark matter searches in the inert doublet model from Peccei-Quinn symmetry, J. High Energy Phys. 10 (2016) 015.

[35] E. Ma, D. Restrepo, and Ó. Zapata, Anomalous leptonic $\mathrm{U}(1)$ symmetry: Syndetic origin of the QCD axion, weakscale dark matter, and radiative neutrino mass, Mod. Phys. Lett. A 33, 1850024 (2018).

[36] S. Chatterjee, A. Das, T. Samui, and M. Sen, A tale of two dark neighbors: WIMP n' axion, arXiv:1810.09471.

[37] S. C. Chuliá, R. Srivastava, and J. W. F. Valle, Seesaw Dirac neutrino mass through dimension-six operators, Phys. Rev. D 98, 035009 (2018).

[38] R. D. Peccei and H. R. Quinn, $C P$ Conservation in the Presence of Instantons, Phys. Rev. Lett. 38, 1440 (1977).

[39] R. N. Mohapatra and G. Senjanovic, The superlight axion and neutrino masses, Z. Phys. C 17, 53 (1983).

[40] Q. Shafi and F. W. Stecker, Implications of a Class of Grand Unified Theories for Large Scale Structure in the Universe, Phys. Rev. Lett. 53, 1292 (1984). 
[41] P. Langacker, R. D. Peccei, and T. Yanagida, Invisible axions and light neutrinos: Are they connected?, Mod. Phys. Lett. A 01, 541 (1986).

[42] M. Shin, Light Neutrino Masses and Strong CP Problem, Phys. Rev. Lett. 59, 2515 (1987); Erratum, Phys. Rev. Lett. 60, 383(E) (1988).

[43] X. G. He and R. R. Volkas, Models featuring spontaneous $C P$ violation: An invisible axion and light neutrino masses, Phys. Lett. B 208, 261 (1988); Radiative generation of quark mixings in a supersymmetric left-right symmetric model, Phys. Lett. B 218, 216 (1989).

[44] Z. G. Berezhiani and M. Yu. Khlopov, Cosmology of spontaneously broken gauge family symmetry, Z. Phys. C 49, 73 (1991).

[45] S. Bertolini and A. Santamaria, The strong $C P$ problem and the solar neutrino puzzle: Are they related?, Nucl. Phys. B357, 222 (1991).

[46] E. Ma, Making neutrinos massive with an axion in supersymmetry, Phys. Lett. B 514, 330 (2001).

[47] C.-S. Chen and L.-H. Tsai, Peccei-Quinn symmetry as the origin of Dirac neutrino masses, Phys. Rev. D 88, 055015 (2013).

[48] S. Bertolini, L. Di Luzio, H. Kolešová, and M. Malinský, Massive neutrinos and invisible axion minimally connected, Phys. Rev. D 91, 055014 (2015).

[49] P.-H. Gu, Peccei-Quinn symmetry for Dirac seesaw and leptogenesis, J. Cosmol. Astropart. Phys. 07 (2016) 004.

[50] E. Ma, T. Ohata, and K. Tsumura, Majoron as the QCD axion in a radiative seesaw model, Phys. Rev. D 96, 075039 (2017).

[51] D. Suematsu, Dark matter stability and one-loop neutrino mass generation based on Peccei-Quinn symmetry, Eur. Phys. J. C 78, 33 (2018).

[52] D. Suematsu, Possible roles of Peccei-Quinn symmetry in an effective low energy model, Phys. Rev. D 96, 115004 (2017).

[53] M. Reig, J. W. F. Valle, and F. Wilczek, $\mathrm{SO}(3)$ family symmetry and axions, Phys. Rev. D 98, 095008 (2018).

[54] M. Reig and R. Srivastava, Spontaneous proton decay and the origin of Peccei-Quinn symmetry, Phys. Lett. B 790, 134 (2019).

[55] J. E. Kim, Weak Interaction Singlet and Strong $C P$ Invariance, Phys. Rev. Lett. 43, 103 (1979).

[56] M. A. Shifman, A. I. Vainshtein, and V. I. Zakharov, Can confinement ensure natural $C P$ invariance of strong interactions?, Nucl. Phys. B166, 493 (1980).

[57] M. Dine, W. Fischler, and M. Srednicki, A simple solution to the strong $C P$ problem with a harmless axion, Phys. Lett. 104B, 199 (1981).

[58] A. R. Zhitnitsky, On possible suppression of the axion hadron interactions (in Russian), Yad. Fiz. 31, 497 (1980) [Sov. J. Nucl. Phys. 31, 260 (1980)].

[59] S. Weinberg, A New Light Boson?, Phys. Rev. Lett. 40, 223 (1978).

[60] F. Wilczek, Problem of Strong p and t Invariance in the Presence of Instantons, Phys. Rev. Lett. 40, 279 (1978).

[61] G. G. Raffelt, Astrophysical axion bounds, Lect. Notes Phys. 741, 51 (2008).
[62] A. Arvanitaki, M. Baryakhtar, and X. Huang, Discovering the QCD axion with black holes and gravitational waves, Phys. Rev. D 91, 084011 (2015).

[63] M. Srednicki, Axion couplings to matter. 1. CP conserving parts, Nucl. Phys. B260, 689 (1985).

[64] D. J. E. Marsh, Axion cosmology, Phys. Rep. 643, 1 (2016).

[65] G. G. Raffelt, Astrophysical methods to constrain axions and other novel particle phenomena, Phys. Rep. 198, 1 (1990).

[66] M. Hirsch, R. Srivastava, and J. W. F. Valle, Can one ever prove that neutrinos are Dirac particles?, Phys. Lett. B 781, 302 (2018).

[67] B. Patt and F. Wilczek, Higgs-field portal into hidden sectors, arXiv:hep-ph/0605188.

[68] Y. G. Kim, K. Y. Lee, and S. Shin, Singlet fermionic dark matter, J. High Energy Phys. 05 (2008) 100.

[69] A. Freitas, S. Westhoff, and J. Zupan, Integrating in the Higgs portal to fermion Dark Matter, J. High Energy Phys. 09 (2015) 015.

[70] C. E. Yaguna, Singlet-doublet Dirac dark matter, Phys. Rev. D 92, 115002 (2015).

[71] S. Bhattacharya, N. Sahoo, and N. Sahu, Minimal vectorlike leptonic dark matter and signatures at the LHC, Phys. Rev. D 93, 115040 (2016).

[72] V. Silveira and A. Zee, Scalar phantoms, Phys. Lett. 161B, 136 (1985).

[73] J. McDonald, Gauge singlet scalars as cold dark matter, Phys. Rev. D 50, 3637 (1994).

[74] C. P. Burgess, M. Pospelov, and T. ter Veldhuis, The minimal model of nonbaryonic dark matter: A singlet scalar, Nucl. Phys. B619, 709 (2001).

[75] N. G. Deshpande and E. Ma, Pattern of symmetry breaking with two Higgs doublets, Phys. Rev. D 18, 2574 (1978).

[76] R. Barbieri, L. J. Hall, and V. S. Rychkov, Improved naturalness with a heavy Higgs: An alternative road to LHC physics, Phys. Rev. D 74, 015007 (2006).

[77] M. Cirelli, N. Fornengo, and A. Strumia, Minimal dark matter, Nucl. Phys. B753, 178 (2006).

[78] P. F. Perez, H. H. Patel, M. .J. Ramsey-Musolf, and K. Wang, Triplet scalars and dark matter at the LHC Phys. Rev. D 79, 055024 (2009).

[79] T. Hambye, F. S. Ling, L. L. Honorez, and J. Rocher, Scalar multiplet dark matter, J. High Energy Phys. 07 (2009) 090; Erratum, J. High Energy Phys. 05 (2010) 66.

[80] M. Kadastik, K. Kannike, and M. Raidal, Matter parity as the origin of scalar Dark Matter, Phys. Rev. D 81, 015002 (2010).

[81] M. Kadastik, K. Kannike, and M. Raidal, Dark matter as the signal of grand unification, Phys. Rev. D 80, 085020 (2009); Erratum, Phys. Rev. D 81, 029903(E) (2010).

[82] M. Kakizaki, A. Santa, and O. Seto, Phenomenological signatures of mixed complex scalar WIMP dark matter, Int. J. Mod. Phys. A 32, 1750038 (2017).

[83] X. Liu and L. Bian, Dark matter and electroweak phase transition in the mixed scalar dark matter model, Phys. Rev. D 97, 055028 (2018).

[84] A. Betancur, R. Longas, and O. Zapata, Doublet-triplet dark matter with neutrino masses, Phys. Rev. D 96, 035011 (2017). 
[85] E. Nardi and E. Roulet, Are exotic stable quarks cosmologically allowed?, Phys. Lett. B 245, 105 (1990).

[86] L. F. Abbott and P. Sikivie, A cosmological bound on the invisible axion, Phys. Lett. 120B, 133 (1983).

[87] K. J. Bae, J.-H. Huh, and J. E. Kim, Update of axion CDM energy, J. Cosmol. Astropart. Phys. 09 (2008) 005.

[88] L. Di Luzio, F. Mescia, and E. Nardi, Window for preferred axion models, Phys. Rev. D 96, 075003 (2017).

[89] L. Di Luzio, F. Mescia, and E. Nardi, Redefining the Axion Window, Phys. Rev. Lett. 118, 031801 (2017).

[90] P. W. Graham, I. G. Irastorza, S. K. Lamoreaux, A. Lindner, and K. A. van Bibber, Experimental searches for the axion and axion-like particles, Annu. Rev. Nucl. Part. Sci. 65, 485 (2015).

[91] N. Du et al. (ADMX Collaboration), A Search for Invisible Axion dark matter with the Axion Dark Matter Experiment, Phys. Rev. Lett. 120, 151301 (2018).

[92] B. M. Brubaker et al., First Results from a Microwave Cavity Axion Search at $24 \mu \mathrm{eV}$, Phys. Rev. Lett. 118, 061302 (2017).
[93] D. S. Akerib et al. (LUX Collaboration), First Searches for Axions and Axionlike Particles with the LUX Experiment, Phys. Rev. Lett. 118, 261301 (2017).

[94] C. Fu et al. (PandaX Collaboration), Limits on Axion Couplings from the First 80 Days of Data of the PandaX-II Experiment, Phys. Rev. Lett. 119, 181806 (2017).

[95] A. Betancur and Ó. Zapata, Phenomenology of doublettriplet fermionic dark matter in nonstandard cosmology and multicomponent dark sectors, Phys. Rev. D 98, 095003 (2018).

[96] E. Ma, Verifiable radiative seesaw mechanism of neutrino mass and dark matter, Phys. Rev. D 73, 077301 (2006).

[97] G. 't Hooft, Naturalness, chiral symmetry, and spontaneous chiral symmetry breaking, NATO Sci. Ser. B 59, 135 (1980).

[98] J. Adam et al. (MEG Collaboration), New Constraint on the Existence of the $\mu^{+} \rightarrow e^{+} \gamma$ Decay, Phys. Rev. Lett. 110, 201801 (2013). 